\title{
Diesel exhaust particulates induce neutrophilic lung inflammation by modulating endoplasmic reticulum stress-mediated CXCL1/KC expression in alveolar macrophages
}

Dong Im Kim

Korea Institute of Toxicology

Mi-Kyung Song

Korea Institute of Toxicology

Kyuhong Lee ( $\sim$ khleekit@gmail.com )

\section{Research}

Keywords: diesel exhaust particulate, endoplasmic reticulum stress, neutrophilic lung inflammation, chemokine CXCL1/KC, alveolar macrophages

Posted Date: March 10th, 2020

DOI: https://doi.org/10.21203/rs.3.rs-16643/v1

License: (c) (i) This work is licensed under a Creative Commons Attribution 4.0 International License.

Read Full License 


\section{Abstract}

Background Exposure to particular matter (PM)2.5, including diesel exhaust particulates (DEP), has adverse effects on the respiratory system. Endoplasmic reticulum (ER) abnormalities contribute to respiratory disease pathogenesis such as lung inflammation. However, there is little published research on the relationship between DEP exposure and ER stress in the respiratory immune system and especially the alveolar macrophages (AM). Here, we examined ER stress and inflammatory responses in a DEPinduced murine lung inflammation model and in DEP-stimulated AM.

Results DEP treatment increased relative lung weight and the number of total cells, neutrophils, and lymphocytes in mouse BALF. Histological examinations also showed that DEP exposure induced neutrophilic lung inflammation and increased the number of DEP-pigmented AM. Western blot analysis showed that $\mathrm{BiP}$ and $\mathrm{CHOP}$ were relatively upregulated in DEP-induced mouse lung tissues. DEP caused cell damage, increased intracellular reactive oxygen species (ROS), and upregulated the genes associated with inflammation (tumor necrosis factor-a, interleukin [IL]-1 $\beta$, IL-6, interferon- $\gamma$, and toll-like receptor 4) and with ER stress (bound immunoglobulin protein [BiP], CCAAT/enhancer binding protein-homologous protein [CHOP], sXBP-1, and activating transcription factor 4) in AM. Furthermore, DEP stimulation upregulated the gene encoding the chemokine CXCL1/KC in AM.

Conclusions DEP may contribute to neutrophilic lung inflammation pathogenesis by modulating ER stress-mediated CXCL1/KC expression in alveolar macrophages.

\section{Background}

Air pollution in urban areas is associated with adverse effects on the respiratory system [1,2]. Major air pollutants include particulate matter (PM), nitrogen oxides (NOX such as nitrogen dioxide $\left(\mathrm{NO}_{2}\right)$ ), ozone $\left(\mathrm{O}_{3}\right)$, sulfur dioxide $\left(\mathrm{SO}_{2}\right)$, carbon monoxide $(\mathrm{CO})$, and hydrocarbons $(\mathrm{HC})[3,4]$. PM is a complex mixture of suspended solid and/or liquid organic and inorganic substances. It comprises coarse particles (diameter range: $2.5-10 \mu \mathrm{m}$ (PM10)), fine particles (diameters < $2.5 \mu \mathrm{m}$ (PM2.5)), and ultrafine particles (diameters $<0.1 \mu \mathrm{m}(\mathrm{UF})$ ) $[2,3]$. The physical and biological properties of PM vary with season, region, and source [4,5]. Diesel exhaust particles (DEP) are emitted by diesel vehicles and constitute a major source of PM2.5 [6]. PM2.5 easily penetrate the lung barrier and enter circulation as they are very small. They are considered a major environmental health risk factor $[1,7,8]$. Several studies reported that increased exposure to PM2.5 is associated with high respiratory and cardiovascular disease morbidity and mortality. Zanobetti et al. reported that the prevalence of respiratory diseases increased by $2.07 \%$ and hospitalization rose by $8 \%$ in response to a $10 \mu \mathrm{g} \mathrm{m}^{-3}$ daily increase in PM2.5 [9-11]. A recent study in China showed that when the PM2.5 concentration rose by $10 \mu \mathrm{g} \mathrm{m}^{-3}$, lung cancer mortality increased by $5.2 \%$ [12]. Prior research has already analyzed and identified the association between PM2.5 and respiratory diseases. However, the specific mechanism of the etiological relationship between them has not been fully elucidated. 
The endoplasmic reticulum (ER) is an organelle that participates in the biosynthesis, correct folding, and posttranslational modifications of secretory and membrane proteins in cells [13]. However, oxidative and metabolic stress, glucose starvation, elevated protein synthesis, and unfolded and misfolded protein accumulation in the ER lumen induce ER stress, triggering a cellular unfolded protein response (UPR) [14, 15]. When ER stress is detected, bound immunoglobulin protein (BiP) dissociates from the ERtransmembrane stress sensors PKR-like eukaryotic initiation factor 2a kinase (PERK), inositol-requiring enzyme 1 (IRE1), and activating transcription factor 6 (ATF6) to restore ER homeostasis. However, when these attempts to recover equilibrium fail, programmed cell death is initiated via CCAAT/enhancer binding protein-homologous protein (CHOP) upregulation [16]. Several studies demonstrated that ER stress influences the development and progression of respiratory diseases such as asthma, fibrosis, and acute lung injury [17-20]. Though ER stress is involved in the pathogenesis of various respiratory disorders, its role in DEP-induced lung inflammation is unclear.

In this study, we investigated the roles and mechanisms of ER stress in DEP-induced lung inflammation by analyzing the cellular changes in BALF, the histological changes, and the ER stress marker (BiP and $\mathrm{CHOP}$ ) expression levels in the lung tissues of DEP-treated mice. We also evaluated cytotoxicity, oxidative stress, and inflammation- and ER stress-related gene expression in DEP-stimulated AM.

\section{Results}

\subsection{Changes in body and lung weights}

The body weights remained constant throughout the experimental period and did not differ among groups (Fig. 2A). After 9 d, the relative mouse lung weights (Fig. 2B) gradually increased in a DEP dosedependent manner and were significantly higher for the DEP 50 and DEP 100 groups than the vehicle control.

\subsection{DEP induces neutrophilic lung inflammation in mice}

DEP exposure adversely affects the respiratory system and is a risk factor for respiratory mortality [1-3]. To determine whether DEP induces an inflammatory response in mouse lungs, we identified and enumerated the number of inflammatory cells in the BALF of DEP-treated mice. The numbers of total cells, macrophages, neutrophils, and lymphocytes in the BALF gradually increased with DEP concentration relative to those in the BALF of the mice in the vehicle control group (Fig. 3A). The inflammatory cells in the BALF of the DEP 100 group were significantly higher than those in the BALF vehicle control group mice. Diff-Quik BALF cell staining (Fig. 3A) and lung tissue H\&E staining (Fig. 3B) showed that DEP exposure induced the accumulation of black, particle-laden AM and the infiltration of inflammatory cells in the peribronchiolar, perivascular, and interstitial regions. These results were confirmed by histological scoring of the infiltrated AMs and inflammatory cells (Table 2). Thus, DEP exposure induces neutrophilic lung inflammation in mice and AM might play a crucial role in this disease process. 


\subsection{DEP initiates ER stress in DEP-treated mice}

To investigate whether DEP exposure is associated with ER stress, we used western blotting to measure the BiP and CHOP protein expression in DEP-treated mouse lung tissues. ER stress markers were significantly higher in the DEP 100 group lung tissues than those of the others (Fig. 4).

\subsection{DEP exposure induces ER stress and activates the UPR pathway in AM}

We confirmed the ER stress markers and the UPR pathway (including sXBP-1 and ATF4) in DEPstimulated AM by RT-qPCR. Relative to the control, the ER stress and UPR pathway-related genes were gradually upregulated in the DEP-stimulated AM. The expression levels of the aforementioned genes were significantly higher in the $3 \mathrm{mg} \mathrm{mL}^{-1}$ DEP AM than the untreated control AM (Fig. 5).

\subsection{DEP-stimulated AM present with inflammatory responses}

We investigated whether DEP exposure induces inflammatory responses in DEP-stimulated AM. RT-qPCR showed that DEP upregulated the inflammatory genes TNF- $a, \mathrm{IL}-1 \beta, \mathrm{IL}-6, \mathrm{KC}$, IFN- $\gamma$, and TLR4 in AM relative to the control group (Fig. 6). The IL-6, KC, IFN-y, and TLR4 mRNA levels were significantly higher in the DEP-stimulated AM than the control (unchallenged) AM.

\subsection{Effects of cell viability and oxidative stress on DEP stimulation in AM}

DEP or PM exposure induces cell damage via ROS-mediated oxidative stress [5, 21, 24]. To determine whether oxidative stress mediates DEP-induced ER stress and inflammatory responses, we evaluated cytotoxicity and reactive oxygen species (ROS) production via MTT assay and DCF-DA fluorescence intensity measurement, respectively. Compared to the untreated control, DEP lowered cell viability and gradually increased ROS production in the AM. Cytotoxicity was accompanied by significant ROS production in AM challenged with $3 \mathrm{mg} \mathrm{mL}^{-1}$ DEP (Fig. 7).

\section{Discussion}

PM is formed mainly by industrial processes and vehicular traffic sources (gasoline and DEP), cooking (coal and oil fuel combustion), and farming and road construction activities [1-4]. DEP are a major source of atmospheric PM2.5 pollution in urban areas and adversely affect human health. Numerous researchers have investigated the associations between air pollution and health risk. In our previous study, a DEP-treated murine model and DEP-stimulated AM demonstrated that DEP induce lung inflammation by modulating the NF-kB signaling pathway [21]. However, the molecular mechanisms involved are still not completely understood.

The endoplasmic reticulum (ER) is a specialized organelle that plays crucial roles in protein biosynthesis, protein folding, and posttranslational secretory and membrane protein alterations. ER stress is triggered by certain conditions such as accumulation of unfolded/misfolded proteins in the ER lumen [14]. Cells initiate an adaptive UPR to restore and maintain ER homeostasis. The four reactions involved in UPR, 
including stimulation of protein degradation, inhibition of translation, production of chaperones, and cell death, are initiated and activated by the transmembrane protein-mediated signaling pathways IRE1, PERK, and ATF6 [25-29]. Several studies demonstrated that ER stress and UPR activation are associated with human pathogenesis and especially inflammation in various metabolic, cardiovascular, and respiratory disorders. Environmental triggers such as cigarette smoking, diesel exhaust, and allergens induce ER stress and UPR in the development and progression of lung diseases including pulmonary fibrosis, asthma, and chronic obstructive pulmonary disease (COPD) [30-36].

To check whether DEP induce lung injury and determine whether ER stress contributes to DEP-induced lung injury in vivo, we assessed the cellular changes in BALF and histological change in lung tissues and measured the ER stress marker (BiP and CHOP) expression levels in lung tissues from DEP-treated mice. The numbers of inflammatory macrophages and neutrophils gradually increased with DEP concentration in the BALF of DEP-treated mice. Histological examination showed that that the number of neutrophils and the quantity of DEP-pigment AM increased in the lungs of DEP-treated mice relative to those of the untreated control. These findings indicate that DEP induce lung inflammation and that AM and neutrophils play important roles in this pathological process. The levels of the ER stress markers BiP and $\mathrm{CHOP}$ were significantly higher in lung tissues presenting with DEP-induced neutrophilic inflammation than they were in the control. Thus, there may have been an interaction between ER stress and neutrophilic lung inflammation pathogenesis in the DEP-treated mice. Therefore, we investigated the association between ER stress and DEP-induced neutrophilic lung inflammation pathogenesis as well as their molecular mechanisms via in vitro DEP stimulation of AM. We evaluated viability, intracellular ROS production, and inflammation- and ER stress-related gene expression in DEP-stimulated AM.

Macrophages are major participants in host defense. As constituents in the innate immune system, they engulf and digest pathogens [37]. In the respiratory system, AM keep the air spaces clear by engulfing foreign materials and minimizing the exposure of other airway cells to these substances [38]. PMpigmented AM participates in inflammatory responses by releasing mediators such as IL-6, IL-8, and tumor necrosis factor (TNF) [24, 39] and producing ROS to kill microorganisms [40, 41]. Excess ROS resulting from redox imbalances induces oxidative stress, is closely associated with ER stress and UPR activation, and initiates inflammatory processes [31, 40-42]. Oxidative stress perturbs the redox environment inside the ER where secretory pathway proteins are produced. Consequently, these proteins are misfolded and trigger ER stress. Conversely, other protein misfolding triggers in the ER provoke excessive ROS production. Therefore, oxidative stress and ER stress are intimately involved in inflammation [15]. Our in vitro study confirmed that DEP stimulation significantly upregulated the ER stress markers BiP and CHOP, the UPR pathway, sXBP-1, and ATF4 in DEP-stimulated AM. Moreover, DEP stimulation increased the mRNA levels of TNF-a, IL-1 $\beta$, IL-6, IFN- $\gamma$, TLR4, and CXCL1/KC in AM. We also used MTT assays and DCF-DA fluorescence intensity measurements to assess whether oxidative stress mediates DEP-induced ER stress and inflammatory responses. Our in vitro study revealed that DEP significantly increased cytotoxicity and oxidative stress in AM relative to the control. Therefore, DEP promote ER stress and induce inflammation by enhancing oxidative stress in AM. 
Several studies demonstrated that after microbial challenges, tissue-resident macrophages are activated to produce neutrophil chemoattractants such as CXCL1, CXCL2, IL-1a, and monocyte chemoattractant protein-1 (MCP-1) [43-45]. They induce rapid neutrophil influx to the infection site. These responses are the result of interactions between neutrophils and macrophages. Chemokine CXCL1/KC plays an important role in inflammation as it is a major chemoattractant responsible for recruiting neutrophils. Zhao et al showed that cellular stress amplifies TLR3/4-induced CXCL1/2 gene transcription in mononuclear phagocytes via RIPK1 [46]. Our result showed that the chemokine gene CXCL1/KC was upregulated in response to DEP stimulation in AM. ER stress-induced neutrophilic inflammation may be a key process in lung disease pathogenesis. Studies $[13,33]$ reported that ER stress contributed to neutrophil inflammation in response to 4-PBA treatment and IL-17A neutralization in animal models of acute lung injury and severe asthma. Thus, our results suggest that chemokine CXCL1/KC released in DEP-stimulated AM could influence the induction and maintenance of ER stress-mediated neutrophilic lung inflammation.

Several researchers have proposed various molecular mechanisms such as enhancement of the proinflammatory factors JNK/AP-1, NF-kB, and PI3K, phosphorylation of PKR, and oxidative stressmediated signaling in ER stress and the UPR pathway associated with lung diseases [30-36]. There are numerous contributing factors connected to the pathogenesis of DEP-induced neutrophilic lung inflammation. Nevertheless, the findings of the present study suggest that DEP might initiate and maintain neutrophilic lung inflammation by upregulating ER stress and UPR-mediated CXCL1/KC expression in AM.

\section{Conclusion}

Our study showed that ER stress markers increased in DEP-induced neutrophilic lung inflammation in vivo. DEP induced ROS production and ER stress- and inflammation-related gene expression in AM. Further research is needed to clarify the mechanisms of these processes. The findings herein suggest that DEP exposure triggers ER stress, activates UPR, increases oxidative stress, damages AM, and induces chemokine CXCL1/KC-mediated neutrophilic lung inflammation.

\section{Methods}

\subsection{Animals}

Female Balb/c mice (Orient Bio, Seongnam, Korea) weighing $16.10 \pm 0.52 \mathrm{~g}$ were housed in a temperature-controlled room $\left(22 \pm 3^{\circ} \mathrm{C}\right)$ under a $12 \mathrm{~h}: 12 \mathrm{~h}$ light/dark cycle, had ad libitum access to standard laboratory chow and tap water, and were acclimated for $7 \mathrm{~d}$ before the experiments. During this time, they presented with normal weight gain and no adverse clinical signs. All animal experiments were performed in accordance with protocols approved by the Institutional Animal Care and Use Committee of the Korea Institute of Toxicology (No. 1711-0434). The Pristima System (v. 7.3; Xybion Medical Systems 
Corp., Morris Plains, NJ, USA) was used to assign the mice randomly into five weight-matched experimental groups ( $n=5$ per group): naive control, vehicle control, DEP 25, DEP 50, and DEP 100.

\subsection{DEP instillation}

The mice in the naïve control group received no treatment for the entire experiment. The mice in the vehicle control group received $50 \mu \mathrm{L}$ saline containing $0.05 \%(\mathrm{v} / \mathrm{v})$ Tween 80 (Sigma-Aldrich Corp., St. Louis, MO, USA). The mice in the DEP 25, DEP 50, and DEP 100 groups were intratracheally instilled with $25 \mu \mathrm{g}, 50 \mu \mathrm{g}$, and $100 \mu \mathrm{g}$ DEP (SRM 2975; National Institute of Standards and Technology, Gaithersburg, MD, USA) dispersed in $50 \mu \mathrm{L}$ vehicle on days 1, 4, and 7, respectively [21].

\subsection{Body and lung weight measurements}

The body weight of mice was measured on days $0,1,4,7$, and 9 . On day 9 , the mice were sacrificed and their lungs were weighed.

\subsection{BALF preparation}

At $48 \mathrm{~h}$ after the last DEP instillation, the mice were anesthetized with isoflurane and exsanguinated. Their left lungs were ligated and their right lungs were gently lavaged thrice via a tracheal tube using a total volume of $0.7 \mathrm{~mL}$ phosphate-buffered saline (PBS). The cells in the BALF were counted with a NucleoCounter (NC-250; ChemoMetec, Gydevang, Denmark). To differentiate the cell types, BALF cell smears were prepared with Cytospin (Thermo Fisher Scientific, Waltham, MA, USA) and stained with DiffQuik solution (Dade Diagnostics, Aguada, Puerto Rico). Two hundred cells per slide were counted.

\subsection{Histological analysis}

On day 9 after the first DEP administration, the mice were sacrificed for histological analysis. Lung tissues were excised, fixed in $10 \%(\mathrm{v} / \mathrm{v})$ neutral-buffered formalin, dehydrated, embedded in paraffin, sliced into 4- $\mu \mathrm{m}$ sections, deparaffinized with xylene, stained with hematoxylin and eosin (H\&E; SigmaAldrich Corp., St. Louis, MO, USA), and viewed under a light microscope (Axio Imager M1; Carl Zeiss AG, Oberkochen, Germany). The degree of inflammation was rated on a $0-4$ scale as previously described $[22,23]$.

\subsection{Protein extract preparation and western blot analysis}

Lung tissues were homogenized and lysed in radioimmunoprecipitation (RIPA) buffer (Thermo Fisher Scientific, Waltham, MA, USA) with a protease inhibitor cocktail. The protein concentrations were determined with Bradford reagent (Bio-Rad Laboratories, Hercules, CA, USA). The samples were loaded onto SDS-PAGE gel. After electrophoresis at $120 \mathrm{~V}$ for $90 \mathrm{~min}$, the proteins were wet-transferred to polyvinylidene difluoride (PVDF) membranes (Bio-Rad Laboratories, Hercules, CA, USA) at $250 \mathrm{~mA}$ for 90 min. Nonspecific sites were blocked with $5 \%(\mathrm{w} / \mathrm{v})$ nonfat dry milk in Tris-buffered saline plus Tween 20 (25 mM Tris ( $\mathrm{pH} 7.5), 150 \mathrm{mM} \mathrm{NaCl}$, and $0.1 \%(\mathrm{v} / \mathrm{v})$ Tween 20$)$ for $1 \mathrm{~h}$ and the blots were incubated 
overnight at $4{ }^{\circ} \mathrm{C}$ with anti-BiP (Cell Signaling Technology, Beverly, MA, USA), anti-CHOP (Cell Signaling Technology, Beverly, MA, USA), and anti-actin (Santa Cruz Biotechnology, Dallas, TX, USA) antibodies. The latter was a reference protein. Anti-rabbit or anti-mouse horseradish peroxidase-conjugated immunoglobulin G (Cell Signaling Technology, Beverly, MA, USA) was used to detect antibody binding. Specific antibody binding was visualized with an iBright CL1000 imaging system (Thermo Fisher Scientific, Waltham, MA, USA) after treatment with enhanced chemiluminescence (ECL) system reagents (Thermo Fisher Scientific, Waltham, MA, USA). Densitometry was performed on each relative band intensity with iBright CL1000 image software (Thermo Fisher Scientific, Waltham, MA, USA). To quantify specific bands, squares of the same size were drawn around all bands, the densities were measured, and the values were adjusted according to the background densities near the bands. The densitometry data were expressed as the relative ratios of the target proteins to the reference protein. The relative ratios of the target control group proteins were arbitrarily set to unity.

\subsection{Murine alveolar macrophage (AM) culture and DEP treatment}

The MH-S murine AM cell line (CRL-2019) was purchased from the American Type Culture Collection (Manassas, VA, USA) and maintained in Roswell Park Memorial Institute (RPMI) 1640 medium (Gibco, Grand Island, NY, USA) supplemented with $10 \%(\mathrm{v} / \mathrm{v})$ fetal bovine serum (FBS) and $1 \%(\mathrm{w} / \mathrm{v})$ penicillinstreptomycin solution (Gibco, Grand Island, NY, USA). The cells were incubated at $37^{\circ} \mathrm{C}$ under a humidified $5 \% \mathrm{CO}_{2}$ atmosphere and treated with DEP $\left(1 \mathrm{mg} \mathrm{mL}^{-1}, 2 \mathrm{mg} \mathrm{mL}^{-1}\right.$, and $\left.3 \mathrm{mg} \mathrm{mL}^{-1}\right)$. After $3 \mathrm{~h}$, the cells were washed twice with PBS and harvested.

\subsection{Cell viability and reactive oxygen species (ROS) measurements}

DEP cell viability and ROS production were evaluated with 3-(4,5 dimethylthiazol-2-thiazyl)-2,5-diphenyltetrazolium bromide (MTT; Sigma-Aldrich Corp., St. Louis, MO, USA) and 3.3 $\mu \mathrm{M}$ 2',7'-dichlorofluorescein diacetate (DCF-DA) (Thermo Fisher Scientific, Waltham, MA, USA), respectively. For the cell viability determination, after DEP stimulation, a $1 \mathrm{mg} \mathrm{mL}^{-1} \mathrm{MTT}$ solution was added to the cells and they were then incubated at $37^{\circ} \mathrm{C}$ for $3 \mathrm{~h}$. The supernatant was removed and the formazan crystals were dissolved in $100 \mu \mathrm{L}$ dimethyl sulfoxide (DMSO; Junsei Chemical Co., Tokyo, Japan). Absorbances were measured at $570 \mathrm{~nm}$ and the background control was measured at $690 \mathrm{~nm}$ in a microplate reader (BioTek, Winooski, VT, USA). Cell viabilities were reported as percentages of the optical density values. To determine the ROS levels, the AM were incubated for $30 \mathrm{~min}$ at $37^{\circ} \mathrm{C}$ in complete RPMI 1640 medium containing $3.3 \mu \mathrm{M}$ DCF-DA. The DCF-DA intensities in the cells were immediately measured at $495 \mathrm{~nm}$ (excitation) and 529 $\mathrm{nm}$ (emission) in a microplate reader. The ROS production levels in the cells were represented as percentages of the DCF-DA intensities relative to the cell viabilities in each well. The latter were defined as $100 \%$.

\subsection{RNA extraction and quantitative real-time polymerase chain reaction (RT-qPCR)}

RNA was extracted from the AM with the RNeasy Mini kit (Qiagen, Venlo, Netherlands) according to the manufacturer's protocol and quantified by measuring absorbance at $260 \mathrm{~nm}$. Then $1 \mu \mathrm{g}$ RNA was reverse- 
transcribed to cDNA in an ImProm-II reverse transcription system (Promega, Madison, WI, USA). The cDNA was quantified with a QuantStudio5 Real-Time PCR system and SYBR Green PCR Master Mix (Applied Biosystems, Foster City, CA, USA). The 20- $\mu \mathrm{L}$ reaction mixtures consisted of $10 \mu \mathrm{L}$ SYBR Green PCR Master Mix, $0.5 \mathrm{pmol}$ of each primer, and $0.5 \mu \mathrm{L}$ undiluted first-strand cDNA. The cycling conditions were as follows: $95^{\circ} \mathrm{C}$ for $10 \mathrm{~min}$ followed by 40 cycles of $95^{\circ} \mathrm{C}$ for $15 \mathrm{~s}$ and $59^{\circ} \mathrm{C}$ for $1 \mathrm{~min}$. The cycle threshold method was used to calculate the relative changes in target gene expression in QuantStudio Design \& Analysis v. 1.4 software (Applied Biosystems, Foster City, CA, USA). The target gene expression levels were normalized to that of actin and were expressed as fold changes. The normalized values of the target gene expression levels in the control group were set to unity. The PCR primers are listed in Table 1.

\subsection{Statistical analysis}

The data were statistically processed with SigmaPlot v. 12 software (Systat, San Jose, CA, USA). Data are means \pm SD. Statistical comparisons were performed by one-way ANOVA followed by Dunnett's test. $P<$ 0.05 was considered statistically significant.

\section{Abbreviations}

4-PBA, 4-phenylbutyrate; AM, alveolar macrophages; ANOVA, analysis of variance; ATF4, activating transcription factor 4; ATF6, activating transcription factor 6; BALF, bronchoalveolar lavage fluid; BiP, binding immunoglobulin protein; CCAAT, cytosine-cytosine-adenosine-adenosine-thymidine; CHOP, C/EBP homologous protein; DCF-DA, 2',7'-dichlorofluorescein diacetate; DEP, diesel exhaust particulate; DMSO, dimethyl sulfoxide; ECL, enhanced chemiluminescence; elF2a, eukaryotic translation initiation factor 2A; ER, endoplasmic reticulum; ERAD, endoplasmic-reticulum-associated protein degradation; FBS, fetal bovine serum; H\&E, hematoxylin and eosin; IFN-y, interferon gamma; IgG, immunoglobulin G; IL, interleukin; IRE1, inositol-requiring enzyme 1; ITI, intratracheal injection; JNK/AP-1, c-Jun $N$-terminal kinase/activator protein 1; KC, keratinocyte chemoattractant; MTT, 3-(4,5-dimethylthiazol-2-yl)-2,5diphenyltetrazolium bromide; NF-kB, nuclear factor kappa-light-chain-enhancer of activated B cells; PBS, phosphate-buffered saline; PDVF, polyvinylidene fluoride; PERK, protein kinase R (PKR)-like endoplasmic reticulum kinase; PI3K phosphatidylinositol 3-kinase; PKR, protein kinase R; PM, particulate matter; RIPA, radioimmunoprecipitation assay; ROS, reactive oxygen species; RPMI, Roswell Park Memorial Institute; RT-qPCR, real-time quantitative polymerase chain reaction; S1P, site-1 protease; S2P, site-2 protease; SDSPAGE, sodium dodecyl sulfate polyacrylamide gel electrophoresis; TLR4, Toll-like receptor 4; TNF-a, tumor necrosis factor alpha; UPR, unfolded protein response; XBP-1, X-box binding protein 1

\section{Declarations}

\section{Ethics approval and consent to participate}


Experimental animals were handled under a protocol approved by the Institutional Animal Care and Use Committee of Korea Institute of Toxicology (No. 1711-0434).

\section{Consent for publication}

Not applicable

\section{Availability of data and materials}

The data used to support the findings of this study are available from the corresponding author upon request.

\section{Competing interests}

The authors have no conflict of interest to declare.

\section{Funding}

This work was supported by the Korea Institute of Toxicology (Grant No. KK-2004).

\section{Authors' contributions}

DIK conducted the experiments, performed the analyses, and wrote the manuscript. MKS edited the manuscript. KL interpreted data and edited the manuscript. All authors read and approved the final manuscript.

\section{Acknowledgements}

Not applicable

\section{References}

1. Kurt OK, Zhang J, Pinkerton KE. Pulmonary health effects of air pollution. Curr Opin Pulm Med. 2106;22:138-43. DOI: 1097/MCP.0000000000000248.

2. Chen TM, Gokhale J, Shofer S, Kuschner WG. Outdoor air pollution: Nitrogen dioxide, sulfur dioxide, and carbon monoxide health effects. Am J Med Sci. 2007;333:249-56. DOI: 10.1097/MAJ.0b013e31803b900f.

3. Falcon-Rodriguez Cl, Osornio-Vargas AR, Sada-Ovalle I, Segura-Medina P. Aeroparticles, composition, and lung diseases. Front Immunol. 2016;7:1-9. DOI:3389/fimmu.2016.00003.

4. Jia YY, Wang Q, Liu T. Toxicity research of $\mathrm{PM}_{5}$ compositions in vitro. Int J Environ Res Pub Heal. 2017;14:1-17. DOI: 10.3390/ijerph14030232.

5. Li N, Wang M, Oberley TD, Sempf JM, Nel AE. Comparison of the pro-oxidative and proinflammatory effects of organic diesel exhaust particle chemicals in bronchial epithelial cells and macrophages. $J$ 
Immunol. 2002;169:4531-41. DOI: 10.4049/jimmunol.169.8.4531.

6. Pant P, Shi Z, Pope FD, Harrison RM. Characterization of traffic-related particulate matter emissions in a road tunnel in Birmingham, UK: Trace metals and organic molecular markers. Aero Air Qual Res. 2017;17:117-30. DOI: 10.4209/aaqr.2016.01.0040.

7. Liu NM, Grigg J. Diesel, children and respiratory disease. BMJ Paediatr Open 2018;2:e000210. DOI:1136/bmjpo-2017-000210.

8. Nemmar A, Holme JA, Rosas I, Schwarze PE, Alfaro-Moreno E. Recent advances in particulate matter and nanoparticle toxicology: a review of the in vivo and in vitro studies. Biomed Res Int. 2013;1-22. DOI:1155/2013/279371.

9. Zanobetti A, Franklin M, Koutrakis P, Schwartz J. Fine particulate air pollution and its components in association with cause-specific emergency admissions. Environ Heal. 2009;8:1-12. DOI:1186/1476069X-8-58.

10. Dominici F. et al. Fine particulate air pollution and hospital admission for cardiovascular and respiratory diseases. J Am Med Assoc. 2006;295:1127-34. DOI: 10.1001/jama.295.10.1127.

11. Xing YF, Xu YH, Shi MH, Lian YX. The impact of PM2.5 on the human respiratory system. J Thorac Dis. 2016;8:E69-E74. DOI:3978/j.issn.2072-1439.2016.01.19.

12. Cao Q, Rui G, Liang Y. Study on PM2.5 pollution and the mortality due to lung cancer in China based on geographic weighted regression model. BMC Publ Heal. 2018;18:1-10. DOl:1186/s12889-0185844-4.

13. Kim SR, Kim DI, Kang MR, Lee KS, Park SY, Jeong JS, Lee YC. Endoplasmic reticulum stress influences bronchial asthma pathogenesis by modulating nuclear factor $\mathrm{KB}$ activation. J Allerg Clin Immunol. 2013;132:1397-1408. DOI:1016/j.jaci.2013.08.041.

14. Walter $P$, Ron $D$. The unfolded protein response: from stress pathway to homeostatic regulation. Science 2011;334:1081-6. DOI:1126/science.1209038.

15. Chen AC, Burr L, McGuckin MA. Oxidative and endoplasmic reticulum stress in respiratory disease. Clin Transl Immunol. 2019;7:e1019. DOI:1002/cti2.1019.

16. Hirai KE, de Sousa JR, Silva LM, Junior LBD, Furlaneto IP, Carneiro FRO, de Souza Aarão TL, Sotto MN, Quaresma JAS. Endoplasmic reticulum stress markers and their possible implications in leprosy's pathogenesis. Dis Markers. 2018;1-10. DOI:1155/2018/7067961.

17. Jeong JS, Kim SR, Cho SH, Lee YC. Endoplasmic reticulum stress and allergic diseases. Curr Allergy Asthma Rep. 2017;17:1-11. DOI:1007/s11882-017-0751-9.

18. Oakes SA, Papa FR. The role of endoplasmic reticulum stress in human pathology. Annu Rev Pathol. 2015;10:173-94. DOI:1146/annurev-pathol-012513-104649.

19. Burman A, Tanjore H, Blackwell TS. Endoplasmic reticulum stress in pulmonary fibrosis. Matrix Biol. 2018;68-9:355-65. DOI:1016/j.matbio.2018.03.015.

20. Marciniak SJ. Endoplasmic reticulum stress in lung disease. Eur Respir Rev. 2017;26:1-7. DOI:1183/16000617.0018-2017. 
21. Kim DI, Song MK, Kim SH, Park CY, Lee K. TF-343 alleviates diesel exhaust particulate-induced lung induced lung inflammation via modulation nuclear factor-KB signaling. J Immunol Res. 2019;2019:8315845. DOI:1155/2019/8315845.

22. Renne R, Brix A, Harkema J, Herbert R, Kittel B, Lewis D, March T, Nagano K, Pino M, Rittinghausen $\mathrm{S}$, Rosenbruch $\mathrm{M}$, Tellier $\mathrm{P}$, Wohrmann T. Proliferative and non-proliferative lesions of the rat and mouse respiratory tract. Toxicol Pathol. 2009;37:5S-73S. DOI:1177/0192623309353423.

23. McInness EF. Background lesions in laboratory animals: A color atlas. Edinburgh: Saunders Elsevier; 2012.

24. Imrich A, Ning Y, Lawrence J, Coull B, Gitin E, Knutson M, Kobzik L. Alveolar macrophage cytokine response to air pollution particles: Oxidant mechanisms. Toxicol Appl Pharmacol. 2007;218:256-64. DOI: 10.1016/j.taap.2006.11.033.

25. Tirasophon W, Welihinda AA, Kaufman RJ. A stress response pathway from the endoplasmic reticulum to the nucleus requires a novel bifunctional protein kinase/endoribonuclease (Ire1p) in mammalian cells. Genes Dev. 1998;12:1812-24. DOI: 10.1101/gad.12.12.1812.

26. Iwawaki T, Hosoda A, Okuda T, Kamigori Y, Nomura-Furuwatari C, Kimata Y, Tsuru A, Kohno K. Translational control by the ER transmembrane kinase/ribonuclease IRE1 under ER stress. Nat Cell Biol. 2001;3:158-64. DOI: 10.1038/35055065.

27. Walter $P$, Ron $D$. The unfolded protein response: from stress pathway to homeostatic regulation. Science 2011;334:1081-6. DOI:1126/science.1209038.

28. Tabas I, Ron D. Integrating the mechanisms of apoptosis induced by endoplasmic reticulum stress. Nat Cell Biol. 2011;13:184-90. DOl:1038/ncb0311-184.

29. Bettigole SE, Glimcher LH. Endoplasmic reticulum stress in immunity. Annu Rev Immunol. 2015;33:107-38. DOI: 10.1146/annurev-immunol-032414-112116.

30. Lindholm D, Korhonen L, Eriksson O, Kõks S. Recent insights into the role of unfolded protein response in ER stress in health and disease. Front Cell Dev Biol. 2017;5:1-16. DOI:3389/fcell.2017.00048.

31. Cao SS, Kaufman RJ. Endoplasmic reticulum stress and oxidative stress in cell fate decision and human disease. Antioxid Redox Signal. 2014;21:396-413. DOI: 10.1089/ars.2014.5851.

32. He M, Ichinose T, Yoshida Y, Arashidani K, Yoshida S, Takano H, Sun G, Shibamoto T. Urban PM2.5 exacerbates allergic inflammation in the murine lung via a TLR2/TLR4/MyD88-signaling pathway. Sci Rep. 2017;7:1-9. DOI:1038/s41598-017-11471-y.

33. Kim SR, Kim HJ, Kim DI, Lee KB, Park HJ, Jeong JS, Cho SH, Lee YC. Blockade of interplay between IL-17A and endoplasmic reticulum stress attenuates LPS-induced lung injury. Theranostics 215;5:1343-62. DOI:7150/thno.11685.

34. Kim SR, Lee YC. Endoplasmic reticulum stress and the related signaling networks in severe asthma. Allergy Asthma Immunol Res. 2015;7(2):106-17. DOl:4168/aair.2015.7.2.106.

35. He M, Ichinose T, Yoshida S, Nishikawa M, Mori I, Yanagisawa R, Takano H, Inoue K, Sun G, Shibamoto T. Urban particulate matter in Beijing, China, enhances allergen-induced murine lung 
eosinophilia. Inhal Toxicol. 2010;22(9):709-18. DOI:3109/08958371003631608.

36. Yang L, Zhao D, Ren J, Yang J. Endoplasmic reticulum stress and protein quality control in diabetic cardiomyopathy. Biochim Biophys Acta. 2015;1852:209-19. DOI:1016/j.bbadis.2014.05.006.

37. Ginhoux F, Guilliams M. (2016). Tissue-resident macrophage ontogeny and homeostasis. Immunity 2016;44(3):439-49. DOI:1016/j.immuni.2016.02.024.

38. Rylance J, Fullerton DG, Scriven J, Aljurayyan AN, Mzinza D, Barrett S, Wright AK, Wootton DG, Glennie SJ, Baple K, Knott A, Mortimer K, Russell DG, Heyderman RS, Gordon SB. Household air pollution causes dose-dependent inflammation and altered phagocytosis in human macrophages. Am J Respir Cell Mol Biol. 2015;52:584-93. DOI:1165/rcmb.2014-01880C.

39. Gordon S, Taylor PR. Monocyte and macrophage heterogeneity. Nat Rev Immunol. 2005;5(12):95364. DOI: $10.1038 /$ nri1733.

40. Yamauchi A, Kim C, Li S, Marchal CC, Towe J, Atkinson SJ, Dinauer MC. Rac2-deficient murine macrophages have selective defects in superoxide production and phagocytosis of opsonized particles. J Immunol. 2004;173(10):5971-9. DOI: 10.4049/jimmunol.173.10.5971.

41. Mohan S, Gupta D. Crosstalk of toll-like receptors signaling and Nrf2 pathway for regulation of inflammation. Biomed Pharmacother. 2018;108:1866-78. DOI:1016/j.biopha.2018.10.019.

42. van der Vliet A, Janssen-Heininger YMW, Anathy V. Oxidative stress in chronic lung disease: From mitochondrial dysfunction to dysregulated redox signaling. Mol Aspects Med. 2018;63:59-69. DOI:1016/j.mam.2018.08.001.

43. De Filippo K, Henderson RB, Laschinger M, Hogg N. Neutrophil chemokines KC and macrophageinflammatory Protein-2 are newly synthesized by tissue macrophages using distinct TLR Signaling pathways. J Immunol 180:4308-4315. DOI: 10.4049/jimmunol.180.6.4308.

44. Prame Kumar K, Nicholls AJ, Wong CHY. Partners in crime: neutrophils and monocytes/macrophages in inflammation and disease. Cell and Tissue Research. 2018; 371:551-565. DOI:1007/s00441-0172753-2.

45. Beck-Schimmer B, Schwendener R, Pasch T, Reyes L, Booy C, Schimmer RC. Alveolar macrophages regulate neutrophil recruitment in endotoxin-induced lung injury. 2005; Respir Res 6:61-61. DOI: 1186/1465-9921-6-61

46. Zhao C, Pavicic PG Jr, Datta S, Sun D, Novotny M, Hamilton TA. Cellular stress amplifies TLR3/4induced CXCL1/2 gene transcription in mononuclear phagocytes via RIPK1. J Immunol. 2014;193:879-88. DOI: 10.4049/jimmunol 1303396.

\section{Tables}

Table 1. RT-qPCR primers. 


\begin{tabular}{lll}
\hline Primer name & Forward primer & Reverse primer \\
\hline BiP & TTCAGCCAATTATCAGCAAACTCT & TTTTCTGATGTATCCTCTTCACCAGT \\
CHOP & CCACCACACCTGAAAGCAGAA & AGGTGAAAGGCAGGGACTCA \\
sXBP-1 & CTGAGTCCGAATCAGGTGCAG & GTCCATGGGAAGATGTTCTGG \\
ATF4 & GGGTTCTGTCTTCCACTCCA & AAGCAGCAGAGTCAGGCTTTC \\
TNF- $\alpha$ & ATGAGCACAGAAAGCATGA & AGTAGACAGAAGAGCGTGGT \\
IL-1 $\beta$ & CAACCAACAAGTGATATTCTCCATG & ATCCACACTCTCCAGCTGCA \\
IL-6 & GCTACCAAACTGGATATAATCAGGA & CCAGGTAGCTATGGTACTCCAGAA \\
IFN- $\gamma$ & TTCTTCAGCAACAGCAAGGC & TCAGCAGCGACTCCTTTTCC \\
TLR4 & AAACGGCAACTTGGACCTGA & AGCTTAGCAGCCATGTGTTCCA \\
CXCL1/KC & CGCTCGCTTCTCTGTGCA & ATTTTCTGAACCAAGGGAGCT \\
actin & GGCACCACACCTTCTACAATG & GGGGTGTTGAAGGTCTCAAAC \\
\hline
\end{tabular}

Table 2. Histologic scores for lungs of DEP-induced mice.

\begin{tabular}{lllllll}
\hline Group & & Naive control & Vehicle control & DEP & DEP & DEP \\
& & & 25 & 50 & 100 \\
\hline Accumulation of black particle-laden & Minimal & 0 & 0 & 1 & 0 & 0 \\
\hline alveolar macrophages and black & Mild & 0 & & & \\
\hline particles in alveolar lumen & Moderate & 0 & 0 & 0 & 0 & 0 \\
\hline & Marked & 0 & 0 & 0 & 0 & 3 \\
\hline Inflammatory cell infiltration & Mean \pm SD & 0 & 0 & $1.8 \pm 0.45^{\# \#}$ & $2.6 \pm 0.55^{\# \#}$ & $3.6 \pm 0.55^{\# \#}$ \\
\hline & Minimal & 0 & 0 & 3 & 0 \\
\hline & Mild & 0 & 0 & 0 & 2 & 5 \\
\hline
\end{tabular}

1: minimal; 2: mild; 3: moderate; 4: marked. Data are means \pm SD for five mice per group. DEP, diesel exhaust particulate. ${ }^{\#} P<$ 0.05 or ${ }^{\# \#} P<0.001$ vs. vehicle control.

\section{Figures}


Figure. 1

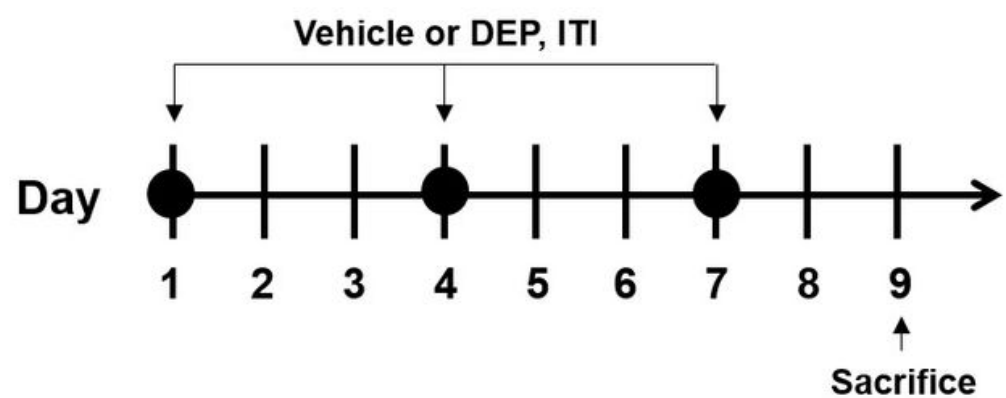

Figure 1

Diagram of in vivo experimental protocol. DEP, diesel exhaust particulate; ITI, intratracheal injection.

Figure 2
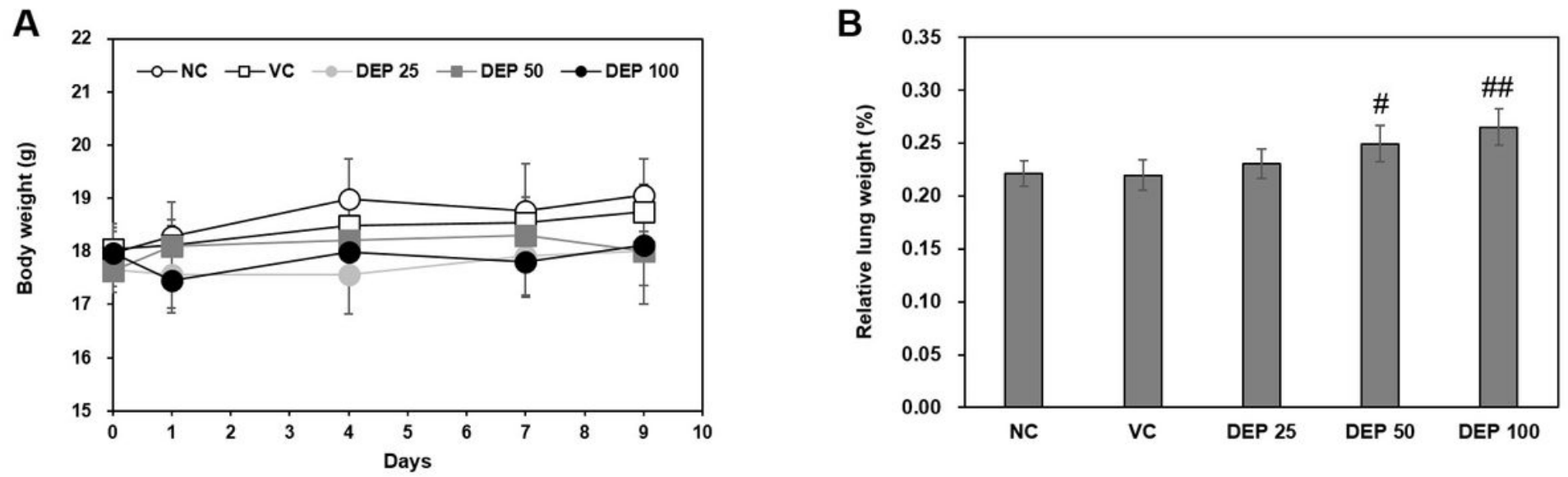

Figure 2

Changes in (A) body weight and (B) relative lung weight in mice in response to DEP instillation. Relative lung weights were calculated as follows: relative organ weight = lung weight $(\mathrm{g}) /$ final body weight $(\mathrm{g}) \times$ $100 \%$. Data are means $\pm S D(n=5$ per group). $\# P<0.05$ or \#\#P<0.01 vs. vehicle control. 


\section{Figure 3}
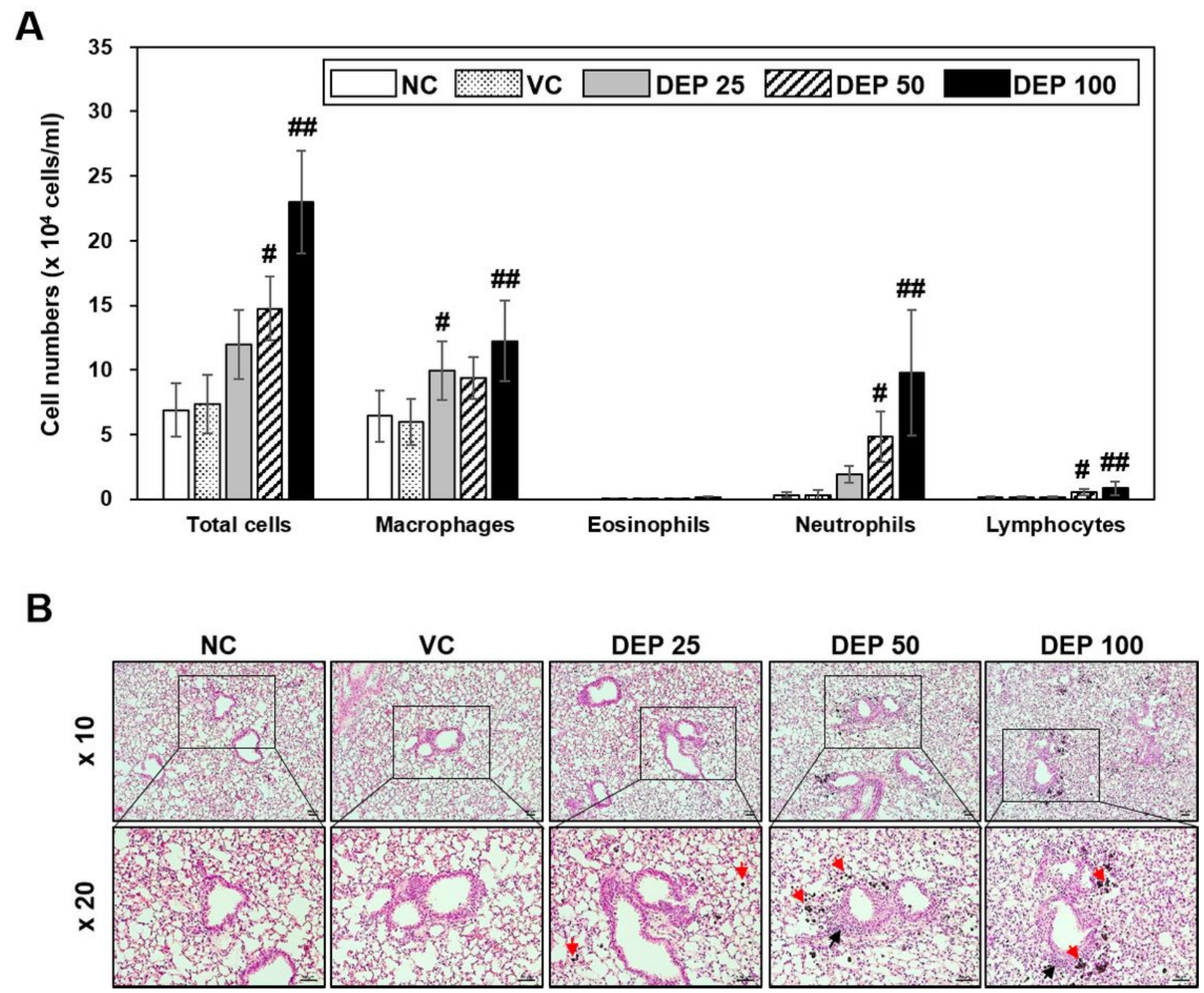

Figure 3

(A) Cellular changes in BALF obtained from naïve control (NC), vehicle control (VC), and DEP $25 \mu \mathrm{g}$ (DEP 25), DEP $50 \mu \mathrm{g}$ (DEP 50), or DEP $100 \mu \mathrm{g}$ (DEP 100) mice. Data are means \pm SD ( $\mathrm{n}=5$ per group). \#P< 0.05 or \#\#P $<0.01$ vs. vehicle control. (B) Histological changes in lung tissue caused by DEP instillation. Representative H\&E-stained sections of lung tissue excised from DEP-induced mice. Red and black arrows indicate black particle-laden alveolar macrophages and inflammatory cells, respectively. Scale bars: $50 \mu \mathrm{m}$. 
Figure 4

A

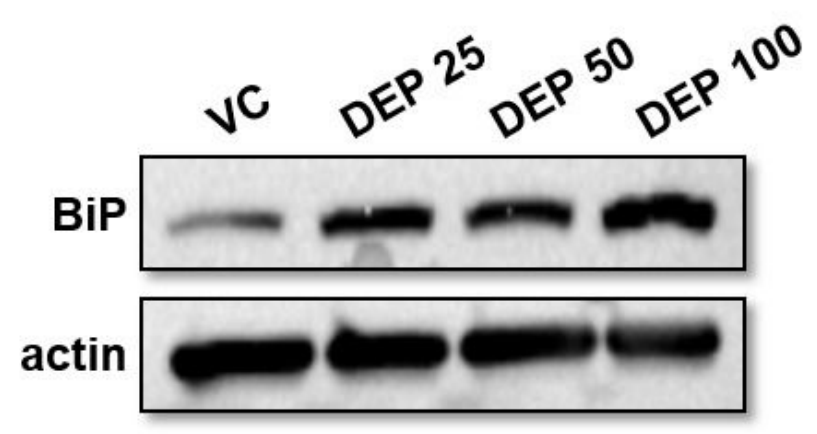

B

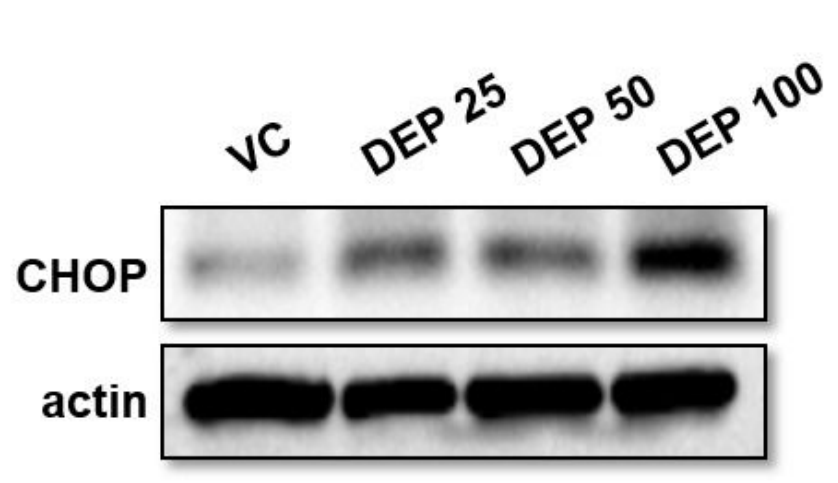

BiP

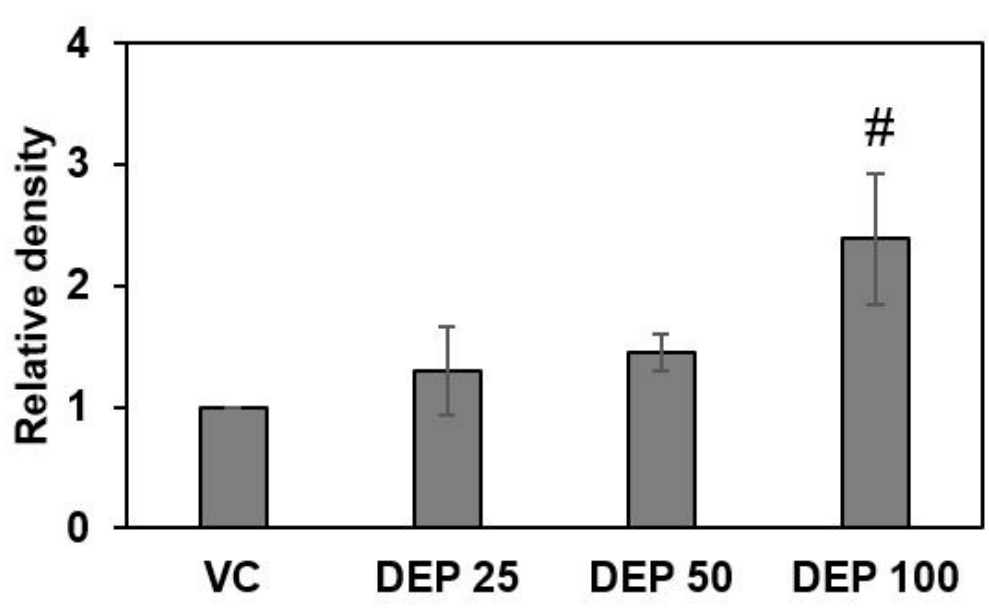

CHOP

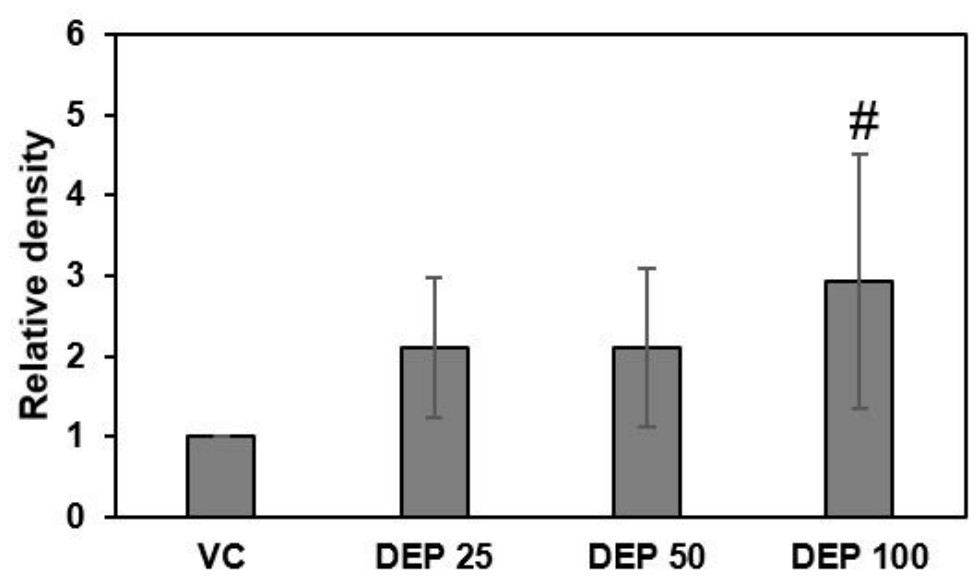

Figure 4

Expression levels of the ER stress markers BiP and CHOP in DEP-induced mice. Representative western blots and relative densities of (A) BiP and (B) CHOP in lung tissues of DEP-induced mice. Data are means $\pm \mathrm{SD}(\mathrm{n}=5$ per group). $\# \mathrm{P}<0.05$ vs vehicle control. 
Figure 5

A

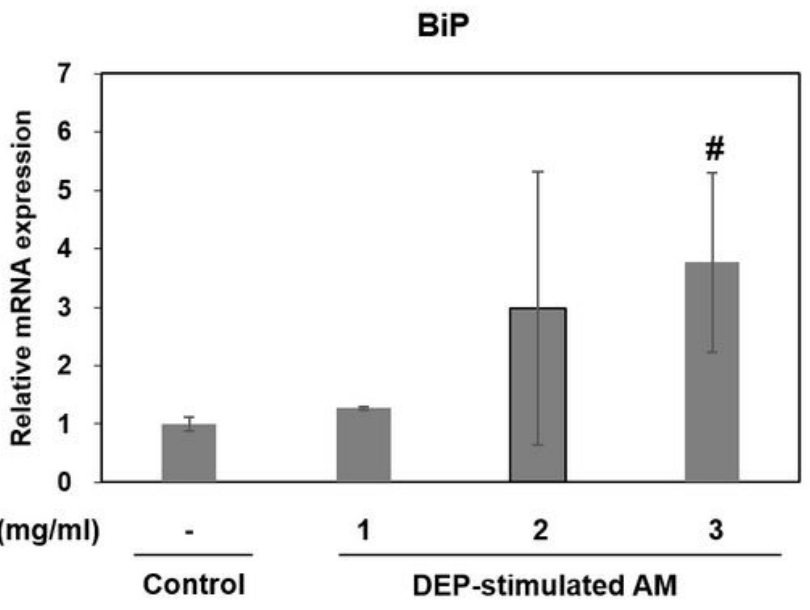

C

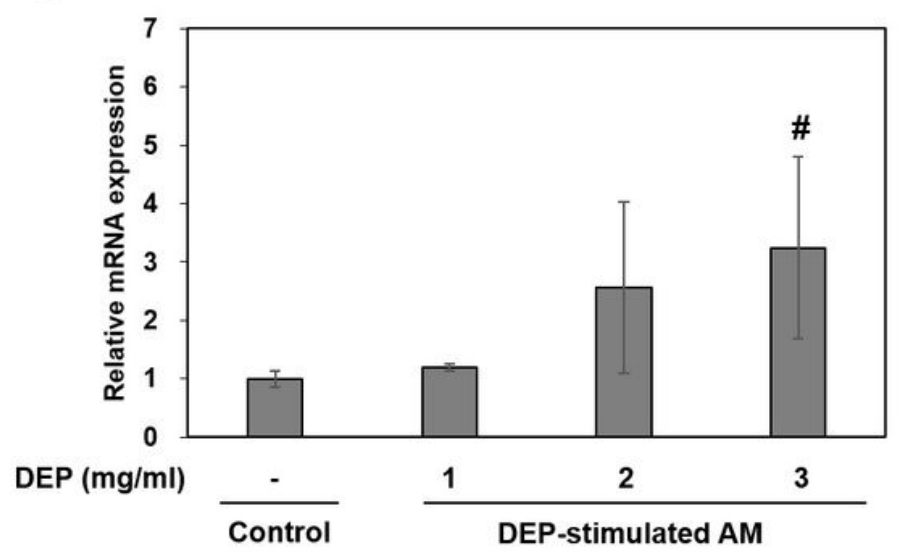

B

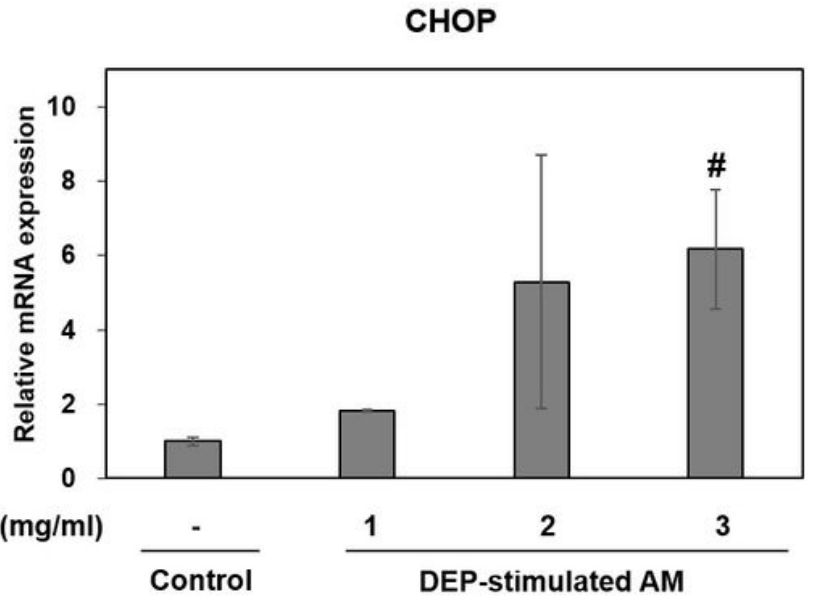

D

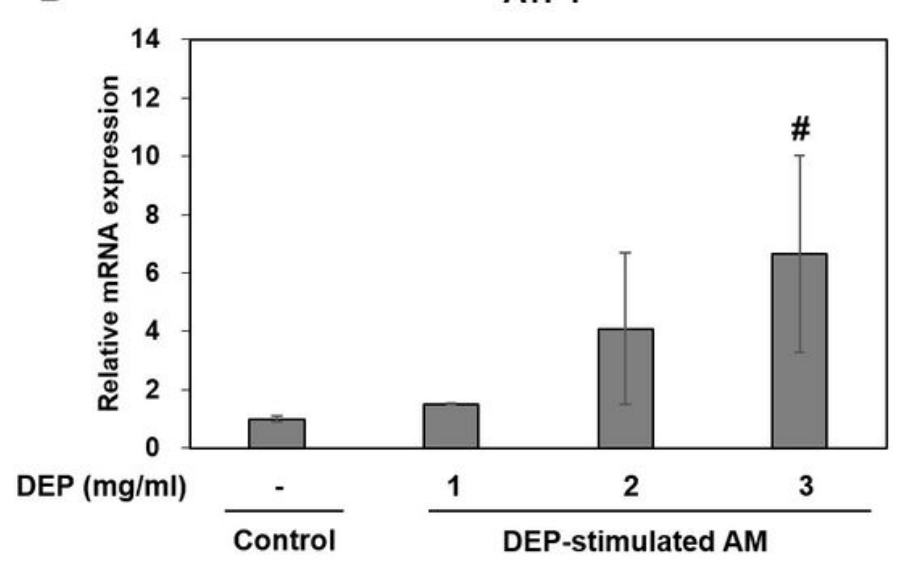

\section{Figure 5}

ER stress-related gene expression levels. (A) BiP, (B) CHOP, (C) sXBP-1, and (D) ATF4 in DEP-stimulated AM. AM were stimulated DEP 1, 2, or $3 \mathrm{mg} \mathrm{mL}-1$ with. RT-qPCR was performed $3 \mathrm{~h}$ after DEP stimulation. Data are means $\pm S D(n=3$ per group). $\# P<0.05$ vs. control. 
Figure 6

A

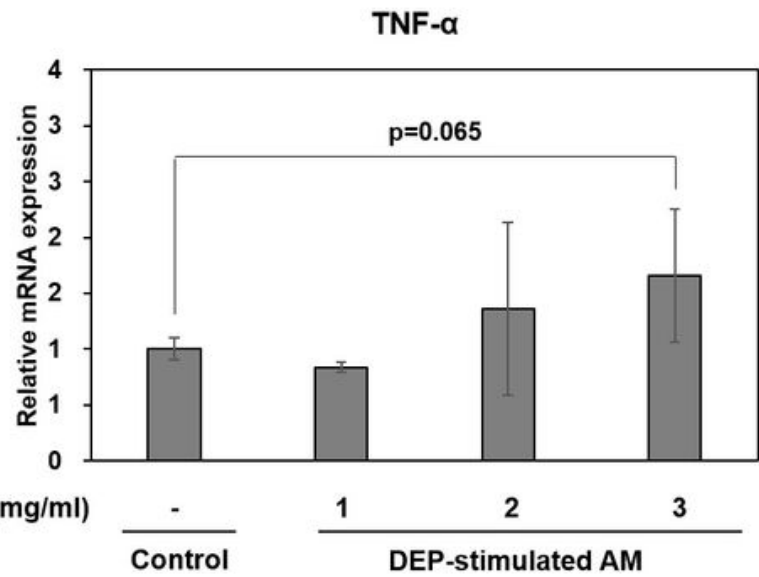

C

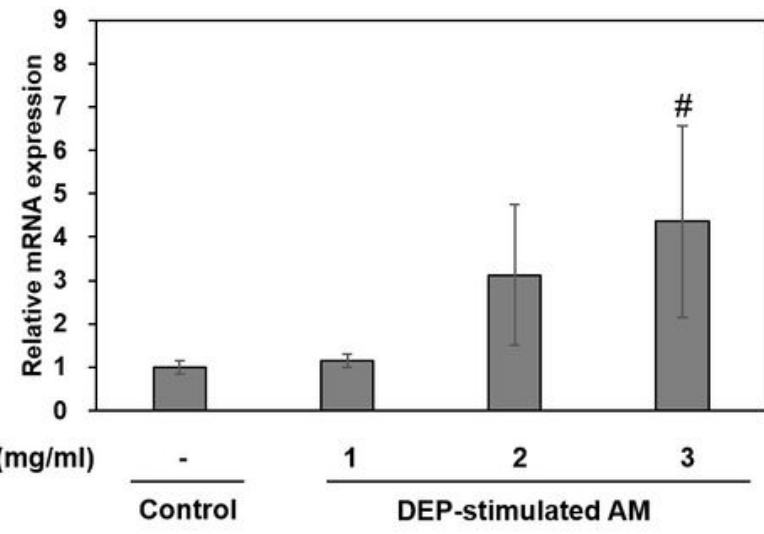

E

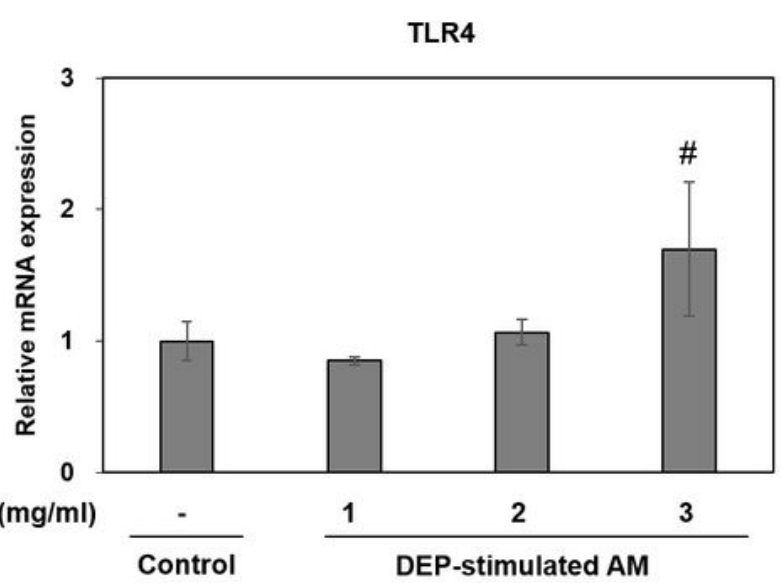

B

IL-1 $\beta$

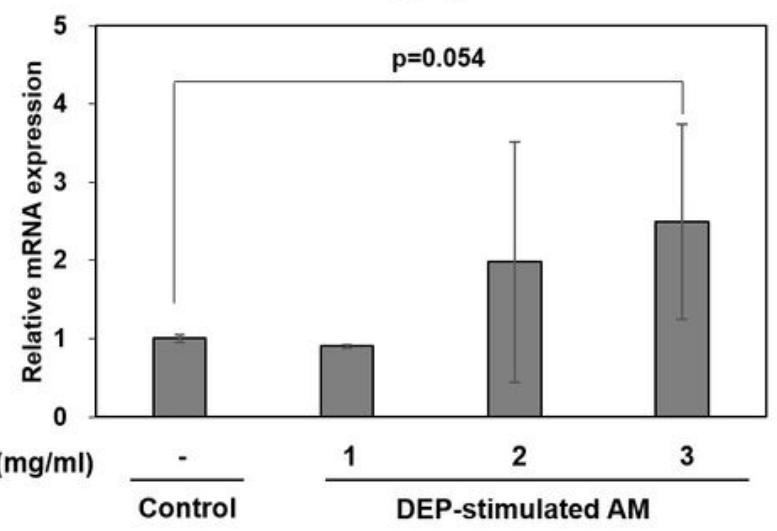

D

IFN-Y

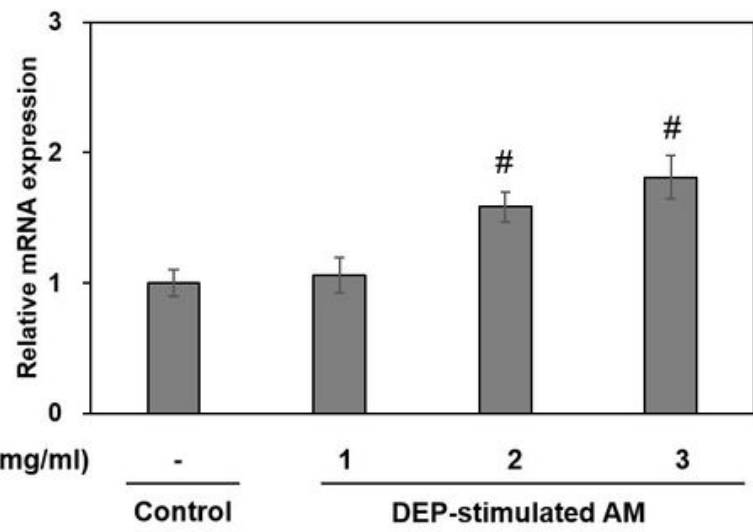

F

CXCL1/KC

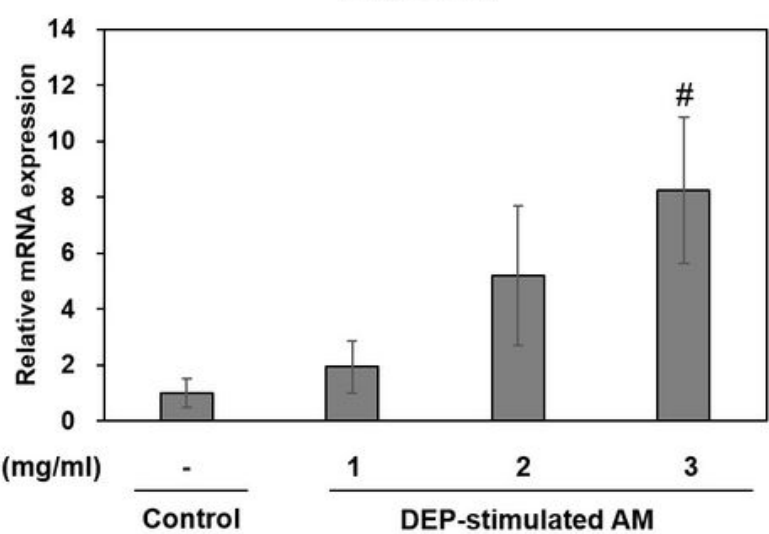

\section{Figure 6}

(A-F) Relative mRNA expression levels for inflammatory factors in DEP-stimulated AM. Data are means \pm SD ( $\mathrm{n}=3$ per group). \#\#P< 0.01 vs. control. 
Figure 7

A

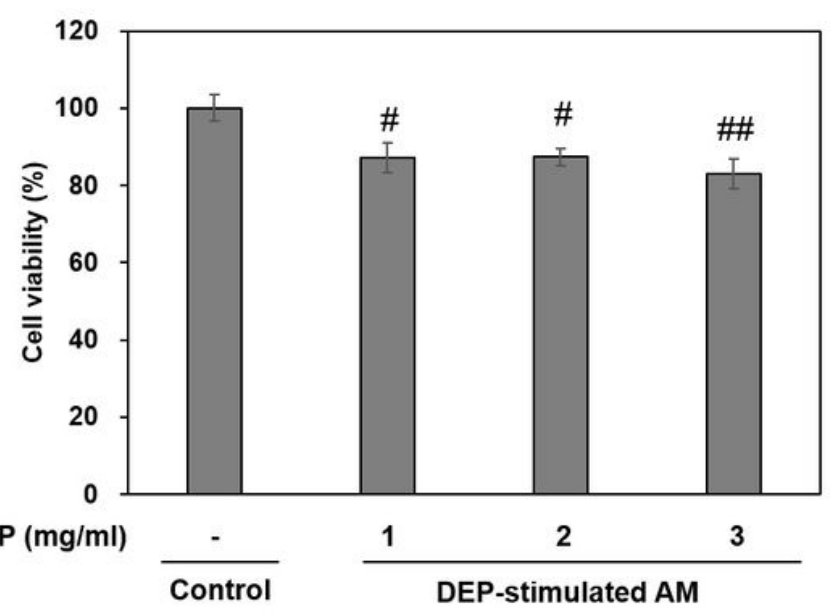

B

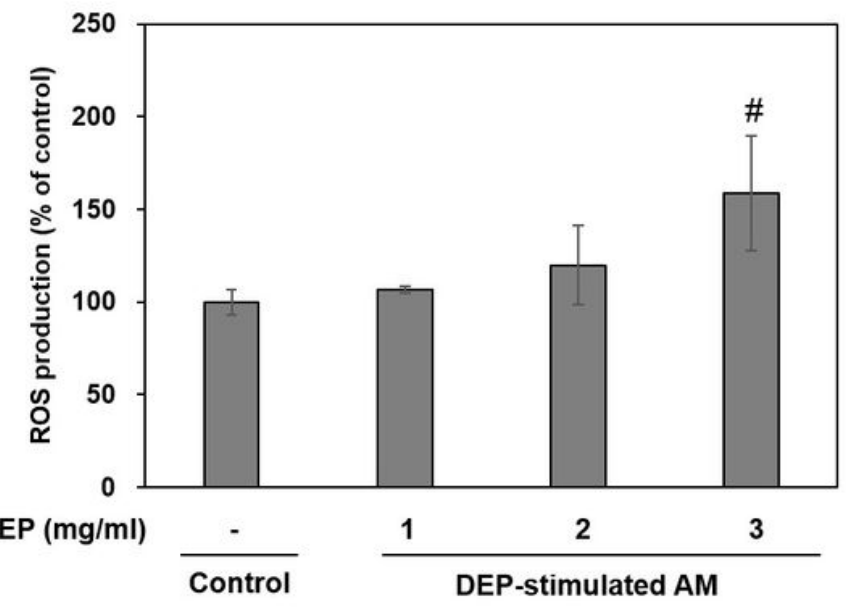

Figure 7

Viability and intracellular ROS production of DEP-treated AM. (A) Cytotoxicity assessment by MTT assay. (B) ROS production measurement by DCF-DA staining. AM were subjected to DEP (1, 2, or $3 \mathrm{mg} \mathrm{mL}-1)$. DCF-DA staining and the MTT assay were performed $3 \mathrm{~h}$ after DEP challenge. Data are means \pm SD $(n=3$ per group). \#P $<0.05$ or \#\#P< 0.01 vs. control. 